\title{
Influenza virus damages the alveolar barrier by disrupting epithelial cell tight junctions
}

\author{
Kirsty R. Shortt, ${ }^{1}$, Jennifer Kasper ${ }^{3}$, Stijn van der Aa ${ }^{1}$, Arno C. Andeweg ${ }^{1}$, \\ Fatiha Zaaraoui-Boutahar ${ }^{1}$, Marco Goeijenbier ${ }^{1}$, Mathilde Richard ${ }^{1}$, \\ Susanne Herold ${ }^{4}$, Christin Becker ${ }^{4}$, Dana P. Scott ${ }^{5}$, Ronald W.A.L. Limpens ${ }^{6}$, \\ Abraham J. Koster ${ }^{6}$, Montserrat Bárcena ${ }^{6}$, Ron A.M. Fouchier ${ }^{1}$, \\ Charles James Kirkpatrick ${ }^{3}$ and Thijs Kuiken ${ }^{1}$
}

\begin{abstract}
Affiliations: ${ }^{1}$ Dept of Viroscience, Erasmus Medical Center, Rotterdam, The Netherlands. ${ }^{2}$ School of Biomedical Sciences, University of Queensland, Brisbane, Australia. ${ }^{3}$ Institute of Pathology, University Medical Center, Johannes Gutenberg University, Mainz, Germany. ${ }^{4}$ University of Giessen and Marburg Lung Center (UGMLC), Justus-Liebig-University of Giessen, Member of the German Center for Lung Research (DZL), Giessen, Germany. ${ }^{5}$ Rocky Mountain Veterinary Branch, Division of Intramural Research, National Institute of Allergy and Infectious Diseases, National Institutes of Health, Hamilton, MT, USA. ${ }^{6}$ Dept of Molecular Cell Biology, Section Electron Microscopy, Leiden University Medical Centre, Leiden, The Netherlands.
\end{abstract}

Correspondence: Thijs Kuiken, Dept of Viroscience, Erasmus Medical Center, Dr Molewaterplein 50, 3015GE Rotterdam, The Netherlands. E-mail: t.kuikenderasmusmc.nl

ABSTRACT A major cause of respiratory failure during influenza A virus (IAV) infection is damage to the epithelial-endothelial barrier of the pulmonary alveolus. Damage to this barrier results in flooding of the alveolar lumen with proteinaceous oedema fluid, erythrocytes and inflammatory cells. To date, the exact roles of pulmonary epithelial and endothelial cells in this process remain unclear.

Here, we used an in vitro co-culture model to understand how IAV damages the pulmonary epithelialendothelial barrier. Human epithelial cells were seeded on the upper half of a transwell membrane while human endothelial cells were seeded on the lower half. These cells were then grown in co-culture and IAV was added to the upper chamber.

We showed that the addition of IAV (H1N1 and H5N1 subtypes) resulted in significant barrier damage. Interestingly, we found that, while endothelial cells mounted a pro-inflammatory/pro-coagulant response to a viral infection in the adjacent epithelial cells, damage to the alveolar epithelial-endothelial barrier occurred independently of endothelial cells. Rather, barrier damage was associated with disruption of tight junctions amongst epithelial cells, and specifically with loss of tight junction protein claudin-4.

Taken together, these data suggest that maintaining epithelial cell integrity is key in reducing pulmonary oedema during IAV infection.

0 @ERSpublications

Influenza A virus damages tight junctions, and specifically claudin-4, of respiratory epithelial cells http://ow.ly/UyGD5

This article has supplementary material available from erj.ersjournals.com

Received: Aug 032015 | Accepted after revision: Nov 032015 | First published online: Jan 072016

Support statement: K.R. Short is supported by a National Health and Medical Research Council (Canberra, Australia) C.J. Martin post-doctoral fellowship (1054081). This study was funded in part by the European Union Seventh Framework Programme project ANTIGONE (grant agreement number 278976), and by the VIRGO consortium, which is funded by the Netherlands Genomics Initiative and by the Dutch Government (project number FES0908). S. Herold is funded by the German Research Foundation (SFB TR84 B2 and SFB 1021 C5) and the German Center for Lung Research (DZL). M. Bárcena is supported by a Netherlands Organisation for Scientific Research (NWO) MEERVOUD-836.10.003 grant. Funding information for this article has been deposited with FundRef.

Conflict of interest: Disclosures can be found alongside the online version of this article at erj.ersjournals.com

Copyright (CERS 2016 


\section{Introduction}

Influenza A virus (IAV) is a respiratory pathogen that causes significant worldwide morbidity and mortality. Typically, IAV causes an acute infection of the upper respiratory tract. However, in severe cases, IAV can cause an infection of the lower respiratory tract, resulting in viral pneumonia and acute respiratory distress syndrome (ARDS). Central to the development of this respiratory dysfunction is damage to the barrier formed by epithelial and endothelial cells in the alveolus. This barrier serves to limit the amount of fluid present in the alveolar airspace, with the epithelial cell component being responsible for the majority of this barrier function [1]. When the epithelial-endothelial barrier becomes damaged, the alveolar airspace can flood with fluid, erythrocytes and leukocytes, ultimately preventing gas exchange and causing severe respiratory insufficiency [2].

At present, how IAV damages the alveolar epithelial-endothelial barrier remains unclear and many different mechanisms have been proposed [2]. For example, alveolar epithelial cells are not only essential in keeping the lung free of fluid but they are also a target cell for IAV infection. IAV-induced epithelial cell death is thus thought to play an important role in barrier dysfunction, and both epithelial cell apoptosis and necrosis have been recorded in patients with IAV-induced ARDS [3, 4]. The apical junction complex (AJC) linking adjacent epithelial cells is also a key part of the fluid barrier formed by the epithelial layer. The AJC consists of tight junction proteins (such as claudins and zona occludens) and adherens junction proteins (such as E-cadherin). By forming at the sites of cell-cell contact, the AJC regulates paracellular permeability and ensures barrier integrity. Several viruses are known to damage the AJC of a wide variety of different cell types [5-9]. However, to the best of our knowledge no study to date has investigated whether IAV can damage the AJC of alveolar epithelial cells.

In addition to epithelial cells, there is a growing body of evidence that endothelial cells also play an important role in IAV-induced oedema and alveolitis [10-12]. For example, in mice, endothelial cells have been identified as the key drivers of the "cytokine storm" observed during severe IAV infections [11]. This excessive cytokine production can either directly (e.g. via the induction of epithelial cell apoptosis) or indirectly (e.g. via the recruitment of leukocytes) damage the epithelial-endothelial barrier [2, 13, 14]. The inflammatory response to IAV may also result in the upregulation of tissue factor, which can be expressed on both endothelial cells and leukocytes. Increased expression of tissue factor on endothelial cells during IAV infection can result in an aberrant pro-coagulant state, leading to the consumption of coagulation factors, microvascular leak and pulmonary haemorrhage [15-17]. Furthermore, a pro-coagulant state induces the release of cytokines such as interleukin (IL)-6 and IL-8 [18], which may further enhance the severity of an IAV-induced cytokine storm and any associated barrier damage. Accordingly, a reduction of the pro-coagulant state of IAV-infected mice is associated with reduced lung injury and improved survival rates [15]. Finally, several in vitro studies have suggested that, like epithelial cells, endothelial cells can be infected by IAV $[8,19-22]$, resulting in cell apoptosis and degradation of the AJC. However, it is important to recognise that, in contrast to infection studies of endothelial cell monolayers in vitro, endothelial cells in vivo are situated below a monolayer of alveolar epithelial cells. Thus, the extent to which endothelial cells get exposed to IAV during in vivo infection remains in question. Indeed, post mortem studies of fatal human IAV cases and of animal models for influenza in humans show that IAV infection of endothelial cells in vivo is either absent or rare [23, 24].

Here, we used an in vitro co-culture model of the alveolar epithelial-endothelial barrier to provide a new insight as to the role of epithelial and endothelial cells in IAV-induced lung damage. We showed that IAV-induced barrier damage is associated with a loss of integrity of the epithelial tight junction, and specifically with a loss of the tight junction protein claudin- 4 .

\section{Materials and methods Cell culture}

$\mathrm{NCl}-\mathrm{H} 441$ cells (which morphologically display characteristics of type II pneumocytes and club cells) $[25,26]$ were obtained from the American Type Culture Collection (Manassas, VA, USA) and cultured in RPMI (Gibco, Grand Island, NY, USA) with 10\% fetal calf serum (FCS) (Sigma, St Louis, MO, USA) and 1\% penicillin-streptomycin (Lonza, Basel, Switzerland). ISO-HAS-1 cells (which have previously been described to display the key features of endothelial cells) [27] were cultured in the same conditions. Primary human pulmonary microvascular endothelial cells (HPMECs) were obtained from Sciencell (Carlsbad, CA, USA) and cultured in endothelial cell growth medium (Sciencell). The co-culture model of the alveolar epithelialendothelial barrier was established essentially as described previously (for full details see the online supplementary material) [25]. Where relevant, cell culture supernatants were treated with sera derived from IAV-vaccinated rabbits, in order to prevent re-infection with IAV. The efficiency of this method in preventing IAV infection was confirmed by flow cytometry (data not shown). 
Viral strains and titrations

Influenza virus strains A/PR8/8/34 (PR8/34; H1N1) and A/Indonesia/05/2005 (Indonesia/05; H5N1) were used to model infection with IAV. Virus stocks were prepared in embryonated chicken eggs (PR8/34) or on Madin Darby Canine Kidney (MDCK) cells (Indonesia/05). Titres of infectious virus were determined as described previously [28].

\section{Viral infection of cell cultures}

$2 \mathrm{~h}$ prior to infection, the medium of the transwell plate was refreshed with RPMI containing $2 \%$ FCS. IAV was then added to the upper compartment of the transwell system. Multiplicity of infection (MOI) was determined based on the average number of epithelial cells present on the transwell membrane prior to infection.

\section{Measurements of barrier integrity}

An EVOM voltohmmeter (World Precision Instruments, Sarasota, FL, USA) with an STX-2 chopstick electrode was used to measure the trans-barrier electrical resistance (TER). Alternatively, permeability to fluorescein isothiocyanate (FITC)-dextran (Sigma) was assessed by adding $2 \mu \mathrm{g}$ of FITC-dextran to the upper compartment of the transwell system. Cells were then incubated for $3 \mathrm{~h}$ at $37^{\circ} \mathrm{C}, 5 \% \mathrm{CO}_{2} .50 \mu \mathrm{L}$ of medium from the lower compartment was then added to $1 \mathrm{mM} \mathrm{NaOH}$ and the fluorescence intensity was measured on a microplate reader (Tecan, Männedorf, Switzerland).

\section{Lactate dehydrogenase release}

Lactate dehydrogenase $(\mathrm{LDH})$ release was determined using the CytoTox 96 Non-Radioactive Cytotoxicity Assay (Promega, Mannheim, Germany) according to the manufacturer's guidelines.

\section{Time to thrombin generation}

Time to thrombin generation was measured essentially as described previously [29]. See the supplementary material for details.

\section{RNA extraction, cDNA synthesis and quantitative PCR}

RNA extraction is described in the supplementary material. cDNA was synthesised using SuperScript III reverse transcriptase (Invitrogen, Carlsbad, CA, USA) and random primers according to the manufacturer's instructions. TaqMan primer/probe combinations (Applied Biosystems, Carlsbad, CA, USA) were used to measure the expression of selected cytokines using glyceraldehyde 3-phosphate dehydrogenase $(\mathrm{GAPDH})$ as a housekeeping gene. Quantitative PCR (qPCR) was performed using the $2 \times$ universal PCR master mix (Applied Biosystems) and the following primer/probe combinations from Applied Biosystems: GAPDH (Hs02758991_g1), tumour necrosis factor (TNF)- $\alpha$ (Hs01113624_g1), chemokine (C-X-C motif) ligand 8 (CXCL8; Hs00174103_m1) and chemokine (C-C motif) ligand 2 (CCL2; Hs00234140_m1). Reactions were performed on a 7500 Real-Time PCR system (Applied Biosystems) and gene expression was calculated using the $2^{-\Delta \Delta \mathrm{Ct}}$ method.

\section{Microarrays}

Preparation and analysis of microarray data is described in the supplementary material.

\section{Electron microscopy}

For electron microscopy, the medium was removed from the upper and lower compartments of a transwell plate at $24 \mathrm{~h}$ post infection. $1.5 \%$ glutaraldehyde in $0.1 \mathrm{M}$ cacodylate buffer $(\mathrm{pH} 7.4$ ) was then added to both compartments in order to fix the cells. After $1 \mathrm{~h}$ at room temperature, the cells were treated with $1 \%$ osmium tetroxide in $0.1 \mathrm{M}$ cacodylate. The cells were then dehydrated in a graded ethanol series. The transwell membrane was cut out and placed onto a plastic flat embedding mould where it was embedded in Epon LX-112 (Ladd Research, Williston, VT, USA). Cross-sections (100 nm) of the cells were cut and collected on carbon-coated pioloform slot grids and post-stained with a saturated aqueous solution of uranyl acetate $(20 \mathrm{~min})$ and lead citrate $(10 \mathrm{~min})$. For imaging information, see the supplementary material. Integrity of the junctions between epithelial cells was measured with the addition of $1 \%$ lanthanum nitrate (Sigma) in the fixative added to the upper compartment. After $5 \mathrm{~h}$ at room temperature, the cells were rinsed and kept overnight with $0.1 \mathrm{M}$ phosphate buffer ( $\mathrm{pH}$ 7.4) to precipitate the lanthanum. The cells were then treated with $1 \%$ osmium tetroxide in $0.1 \mathrm{M}$ cacodylate buffer, which, in the upper compartment, also contained $1 \%$ lanthanum nitrate. Following processing, images were collected at a magnification of $1900 \times$ with binning 2 (corresponding to a pixel size of $11.5 \mathrm{~nm}$ at the specimen level). The permeability of the tight junctions in these images was then assessed by blindly determining the number of lanthanum-positive junctions in mock- and IAV-infected samples. 


\section{Immunofluorescence}

Cells grown on a transwell membrane were fixed with $4 \%$ paraformaldehyde for $\geqslant 20$ min at room temperature and subsequently washed with PBS. Cells were then permeabilised for $15 \mathrm{~min}$ at room temperature with $1 \%$ Triton X (Sigma). Cells were stained with the relevant primary antibody followed by the relevant secondary antibody in PBS/1\% bovine serum albumin (Sigma)/0.5\% Triton $\mathrm{X}$ for $1 \mathrm{~h}$ at room temperature. Primary antibodies used were $\alpha$-influenza A nucleoprotein (HB-65; American Type Culture Collection), $\alpha$-E-cadherin (Bioss Company, Beijing, China), $\alpha$-occludin (Sigma), $\alpha$-junctional adhesion molecule 1 (Santa Cruz Biotechnology, Santa Cruz, CA, USA), $\alpha$-zona occludin 1 and $\alpha$-claudin-4 (both Life Technologies, Gaithersburg, MD, USA). Secondary antibodies were Alexa $488 \alpha$-mouse IgG2a, Alexa $594 \alpha$-mouse IgG and Alexa $488 \alpha$-rabbit IgG (all from Life Technologies). Transwell membranes were then washed three times with PBS, excised and mounted on a glass slide with mounting medium containing 4',6-diamidino-2-phenylindole (DAPI) (Vector Laboratories, Burlingame, CA, USA). Staining was visualised using a Laser Scanning Microscope 700 (LSM 700) (Zeiss, Jena, Germany) and staining intensity was quantified using ImageJ software (National Institutes of Health, Bethesda, MD, USA).

\section{Statistical analysis}

Statistical analyses were performed using GraphPad Prism, version 5.00 for Windows (GraphPad Software, La Jolla, CA, USA).

\section{Results}

IAV damages the alveolar barrier in an in vitro co-culture model

To understand the role of epithelial and endothelial cells in IAV-induced barrier damage, we adapted a previously described model of the alveolar epithelial-endothelial barrier [25]. Briefly, alveolar epithelial cells (NCl-H441 cells) were seeded atop a permeable transwell membrane while endothelial cells (ISO-HAS-1 cells) were seeded directly below the transwell membrane (figure 1). To mimic IAV infection via the respiratory route, $\mathrm{PR} 8 / 34$ was added to the upper compartment and barrier integrity was measured over time. Infection with IAV at an MOI of 1 or 0.2 resulted in a significant decline in the TER compared with mock-infected cells at $24 \mathrm{~h}$ post-infection (figure $2 \mathrm{a}$ ). In contrast, there was no significant difference in the TER between mock-treated cells and cells treated with UV-inactivated PR8/34. There was also a significant increase in permeability to FITC-labelled dextran following IAV infection relative to mock-infected cells (figure 2b). Together, these data demonstrate that IAV infection is able to damage this in vitro epithelial-endothelial barrier.

To determine the cell tropism of IAV infection, the co-cultured epithelial and endothelial cells were stained for IAV antigen. At $24 \mathrm{~h}$ post-infection at an MOI of 0.2 , epithelial cells were positive for IAV antigen (figure $2 \mathrm{c}$ ), with $\sim 40 \%$ of cells being infected (data not shown). In contrast, no infection could be detected in endothelial cells either by immunofluorescence (figure 2c) or flow cytometry (data not shown), even though low levels of IAV were detected in the medium of the lower compartment of the co-culture (figure $2 \mathrm{~d}$ ). We then sought to determine whether endothelial cells were at all permissive to IAV infection in this system. Accordingly, a monoculture of endothelial cells was grown below the transwell membrane and IAV was added to the upper compartment. At $24 \mathrm{~h}$ post-infection, endothelial cells stained positive for IAV antigen (figure 2e). These data suggest that, although endothelial cells are permissive for IAV infection, they do not become infected when IAV is inoculated on the epithelial layer of a co-culture, most likely due to the limited number of virus particles that come into contact with endothelial cells.

\section{IAV induces a stronger pro-inflammatory response in epithelial cells than in endothelial cells of an in vitro alveolar barrier}

Previous studies have suggested that endothelial cells are the key drivers of the cytokine storm during IAV infection of mice [11]. Hence, we reasoned that despite not being infected by IAV, endothelial cells may play a role in barrier dysfunction by producing pro-inflammatory cytokines in response to the infection in the adjacent epithelial cells. Due to the permeability of the transwell membrane, cytokines detected in the lower 


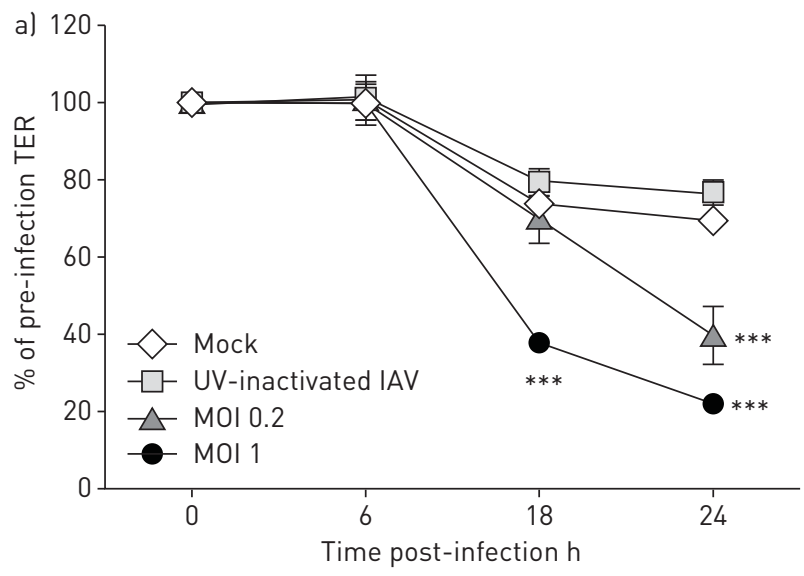

c)

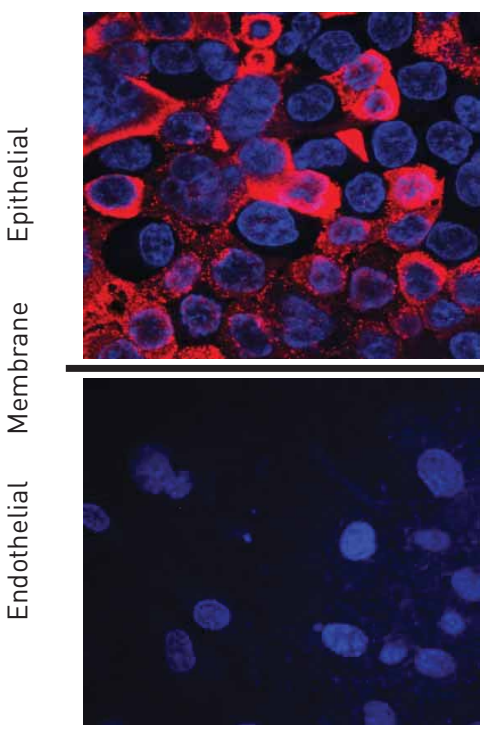

e)

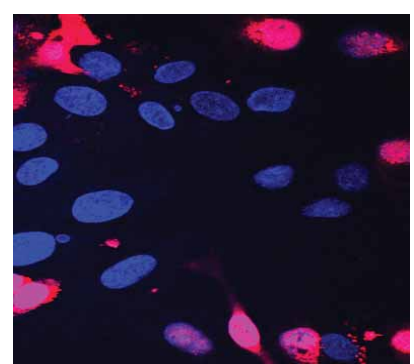

Mock
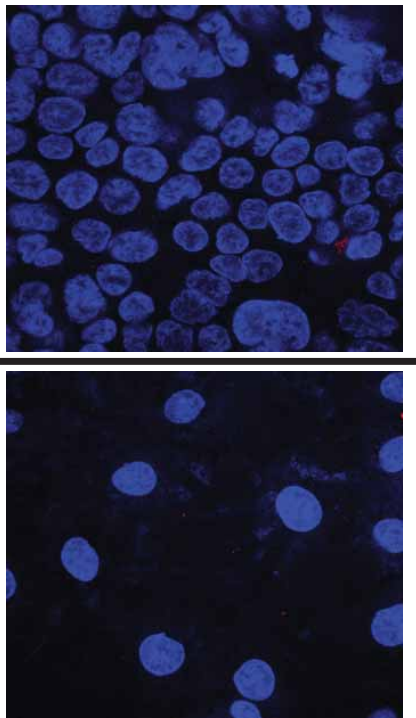

Mock

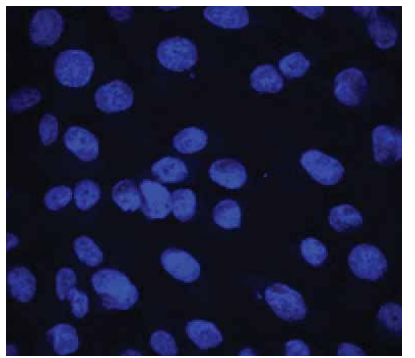

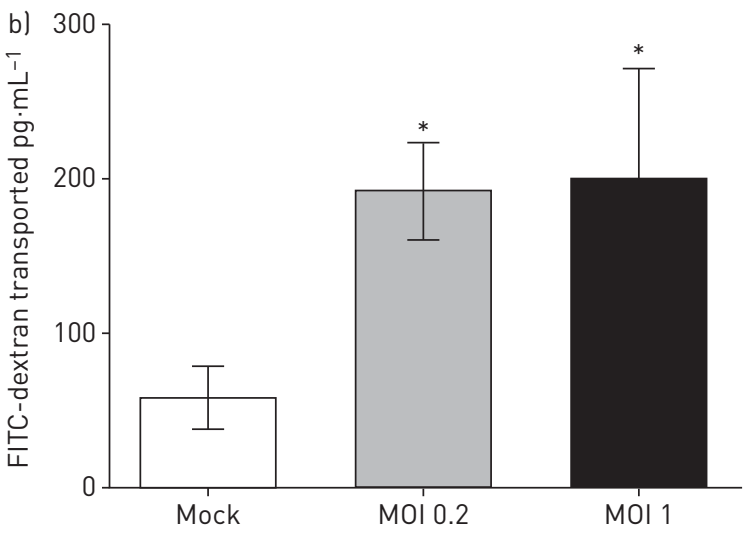

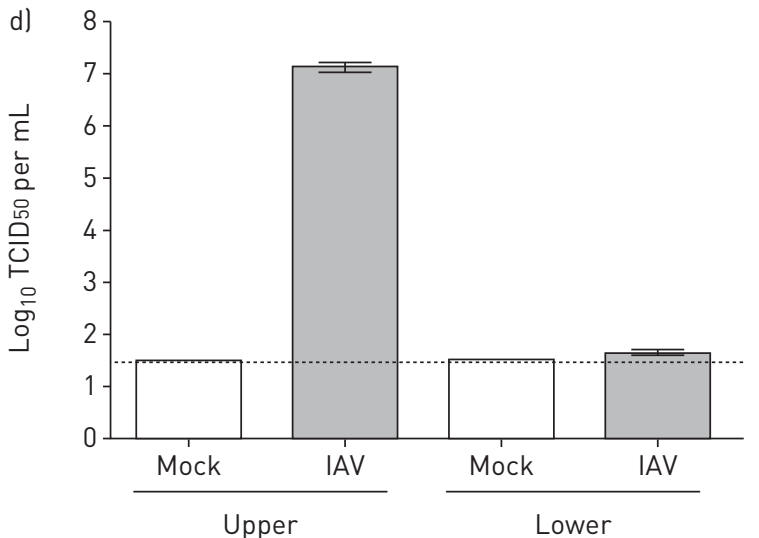

FIGURE 2 Influenza A virus (IAV) induces damage to an in vitro model of the alveolar epithelial-endothelial barrier. a) Measurement of the trans-barrier electrical resistance (TER) of epithelial-endothelial co-cultures over time. Data are expressed relative to the TER recorded just prior to infection (which was defined as 100\%) with mock, UV-inactivated IAV (strain PR8/34 (H1N1)) or IAV (PR8/34) at a multiplicity of infection (MOI) of 0.2 or 1. b) Permeability of epithelial-endothelial co-cultures to fluorescein isothiocyanate (FITC)-dextran. Data show the concentrations of FITC detected in the lower compartment following the addition of $2 \mu \mathrm{g}$ of FITC-dextran to the upper compartment. FITC-dextran was added to the upper compartment at $24 \mathrm{~h}$ post-infection with IAV (PR8/34), and cells were incubated for $3 \mathrm{~h}$ prior to the measurement of FITC in the lower compartment. c) Representative immunofluorescence images of co-culture transwell membranes at $24 \mathrm{~h}$ post-infection with either medium ("mock") or IAV (PR8/34, MOI 0.2). Nuclei are stained blue while the IAV nucleoprotein is stained red. Images are of epithelial and endothelial cells derived from the same membrane. d) Virus titres detected in the supernatants of the upper and lower compartments of a co-culture at $24 \mathrm{~h}$ post-infection with medium ("mock") or IAV (PR8/34, $\mathrm{MOI}$ 0.2). The detection limit of the assay is indicated by a dotted line. TCID50: median tissue culture infective dose. e) Representative immunofluorescence images of transwell membranes with a monoculture of endothelial cells stained for IAV antigen. Cells were infected with the same dose of IAV (PR8/34) as those in c) and images were taken $24 \mathrm{~h}$ post-infection. Nuclei are stained in blue while the IAV nucleoprotein is stained in red. $a, b$ and d) Data are pooled from at least three independent experiments and shown as mean \pm SEM. Statistical significance relative to mock-infected cells was calculated by a) two-way ANOVA or b) one-way ANOVA with Bonferroni's multiple comparison test. *: $p<0.05 ;{ }^{* * *}$ : $p<0.001$. 
compartment are not necessarily produced exclusively by endothelial cells. Thus, in order to assess cytokine production by endothelial cells, epithelial and endothelial cell populations were separated from the transwell membrane. RNA was then extracted and microarray-assisted gene expression profiling was performed on both cell populations. IAV-infected epithelial cells displayed 261 differentially expressed genes relative to mock-infected cells. In contrast, endothelial cells exposed to IAV-infected epithelial cells only displayed 36 differentially expressed genes relative to endothelial cells that were exposed to mock-infected epithelial cells (figure 3). Upregulated cytokine genes in endothelial cells included CXCL10, CCL2, CCL8, CXCL11 and CXCL2. Several other genes associated with an anti-viral response (e.g. 2'-5'-oligoadenylate synthetase) and leukocyte adhesion/diapedesis (e.g. vascular cell adhesion molecule 1) were also differentially expressed. Interestingly, with the exception of CCL8 and CCL2, all cytokine genes that were differentially expressed in endothelial cells were also differentially expressed in epithelial cells (figure 3), and often to higher levels. For example, CCL20 was almost 30-fold upregulated in infected epithelial cells while only being two-fold

Chemokine (C-X-C motif) ligand 10

Chemokine ( $C-C$ motif) ligand 2

Chemokine (C-X-C motif) ligand 11

Nuclear receptor coactivator 7

Lymphotoxin beta (TNF superfamily, member 3)

CD69 molecule

XIAP associated factor 1

Chemokine (C-C motif) ligand 20

Receptor (chemosensory) transporter protein 4

Radical S-adenosyl methionine domain containing 2

Myxovirus resistance 2

Myxovirus resistance 1, interferon-inducible protein p78

Intercellular adhesion molecule 1

Interferon-induced protein with tetratricopeptide repeats 3

Guanylate binding protein 1, interferon-inducible

Rho family GTPase 1

TNF (ligand) superfamily, member 10

Epithelial stromal interaction 1

Interferon regulatory factor 1

Selectin E

Guanylate binding protein 1, interferon-inducible

Chemokine (C-X-C motif) ligand 2

DEAD (Asp-Glu-Ala-Asp) box polypeptide 58

Interferon-induced protein with tetratricopeptide repeats 1

2'-5'-Oligoadenylate synthetase 1, 40/46 kDa

Cytidine monophosphate (UMP-CMP) kinase 2, mitochondrial

Vascular cell adhesion molecule 1

Chemokine (C-C motif) ligand 8

TNF alpha-induced protein 3

Neuralized homolog 3 (Drosophila) pseudogene

Interferon-induced protein with tetratricopeptide repeats 5

Baculoviral IAP repeat containing 3

Transporter 1, ATP-binding cassette, sub-family B (MDR/TAP)

Sterile alpha motif domain containing 9-like

Interferon induced with helicase $\mathrm{C}$ domain 1

TNF receptor-associated factor 1 fold change fold change

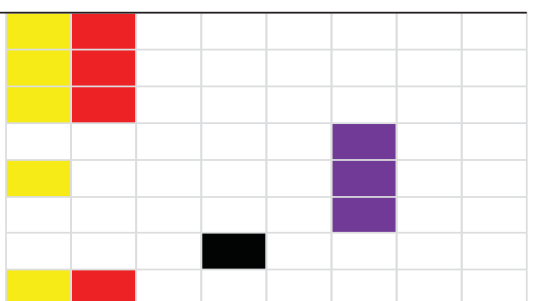

29.82

2.75

2.73

2.72

7.58

4.81

9.06

2.68

2.64

2.64

8.92

2.60

2.54

5.67

2.50

4.10

2.48

2.41

2.75

2.40

2.39

2.09

3.46

2.33

3.79

2.28

2.28

2.24

6.63

2.23

6.98

2.20

2.19

2.19

14.11

2.19

2.18

2.90

2.16

3.20

2.14

4.02

2.09

2.09

3.27

2.06

2.05

FIGURE 3 Gene expression profiles of co-cultured epithelial and endothelial cells following influenza A virus (IAV) infection. Co-cultures were infected with IAV (strain PR8/34 (H1N1), at a multiplicity of infection of 0.2) for $24 \mathrm{~h}$. Epithelial and endothelial cells were then separated from the transwell membrane and used for transcriptional profiling. The 36 genes, and their corresponding fold changes in epithelial and endothelial cells, are shown here. The absence of a fold change value means that differential expression of the relevant gene was not detected in IAV-infected epithelial cells when applying a false discovery rate of 0.05 and a fold change cut-off of 2 . A simple functional classification of the genes of interest is indicated. TNF: tumour necrosis factor; XIAP: X-linked inhibitor of apoptosis; IAP: inhibitor of apoptosis. 
upregulated in endothelial cells. Furthermore, several different cytokines (such as CCL20 and CXCL8) were exclusively differentially expressed in epithelial cells (supplementary figure S1). To confirm these findings, qPCR was subsequently performed on co-cultured epithelial and endothelial cells for two genes that were differentially expressed in epithelial cells (TNF- $\alpha$ and CXCL8) and one gene that was differentially expressed in endothelial cells (CCL2). Consistent with the microarray data, TNF- $\alpha$ and CXCL8 were significantly more upregulated in epithelial cells than in endothelial cells (figure S1a). Surprisingly, CCL2 was upregulated in both cell types, while the microarray data did not detect CCL2 upregulation in infected epithelial cells. Finally, to confirm that some of the selected cytokines were also produced on a protein level, ELISAs were performed on the cell supernatant from the upper and lower compartments of IAV- and mock-infected co-cultures (figure S1b). As expected, statistically significantly higher levels of TNF- $\alpha$ and CXCL8 were detected in the supernatant (upper and lower) of IAV-infected co-cultures compared with those that were mock infected. Together, these data suggest that while endothelial cells mount a pro-inflammatory response to the IAV infection in the adjacent epithelial cells, epithelial cells are the key drivers of the cytokine response in this in vitro system.

\section{IAV infection of epithelial cells results in a pro-coagulant state}

An aberrant pro-coagulant response has been previously described to contribute to IAV pathogenesis $[15,16]$. To determine if an IAV infection in epithelial cells was sufficient to induce a pro-coagulant state in the adjacent endothelial cells, we measured the time to thrombin generation by incubating human plasma with either IAV- or mock-infected co-cultures. IAV-infected co-cultures displayed a significantly reduced time to thrombin generation compared with mock-infected co-cultures (figure 4). Thus, endothelial cells are able to induce a pro-coagulant state, despite not being directly infected by IAV.

\section{Endothelial cells do not contribute to damage of the in vitro alveolar barrier}

While the production of cytokines and a pro-coagulant state may have important consequences in vivo, we wished to determine if these, or other, features of endothelial cells contributed to the barrier damage observed in vitro. To do so, we compared IAV-induced damage between epithelial-endothelial co-cultures and monocultures of epithelial cells. In both situations, IAV significantly decreased the TER over time (figure 5a). However, no significant difference in barrier damage was observed between IAV-infected epithelial cell monocultures and IAV-infected co-cultures (figure 5a). Similarly, there was no significant difference in the permeability of IAV-infected mono- and co-cultures to FITC-labelled dextran (figure 5b). To confirm that these observations were not an artefact of the use of immortalised endothelial cells, or of the model IAV strain used, we established a co-culture of epithelial cells and primary endothelial cells (HPMECs) as well as a monoculture of epithelial cells. These cultures were then infected with the highly pathogenic avian influenza virus Indonesia/05. Consistent with our previous observations with PR8/34 and co-cultures using immortalised endothelial cells, infection with Indonesia/05 significantly decreased the TER of epithelial-HPMEC co-cultures and epithelial monocultures over time (figure $5 \mathrm{c}$ ). However, there was no significant difference in TER observed between infected co-cultures and infected monocultures

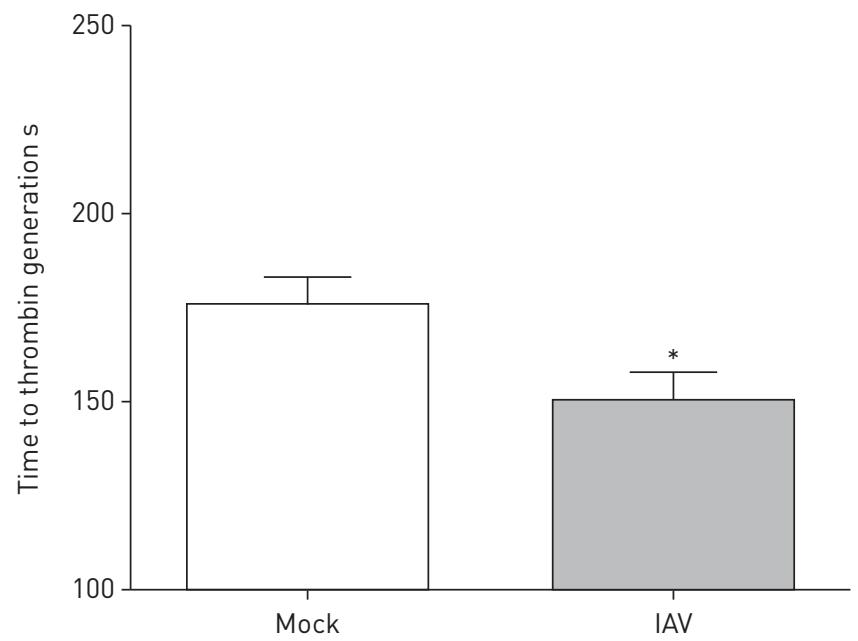

FIGURE 4 Time to thrombin generation of mock-infected or influenza A virus (IAV)-infected co-cultures. IAV (strain PR8/34 (H1N1)) was infected at a multiplicity of infection of 0.2. Co-cultures were incubated with human plasma and the time to thrombin generation was measured. Data are pooled from three independent experiments and shown as mean士SEM. Statistical significance relative to mock-infected cells was calculated by t-test. *: $p<0.05$. 

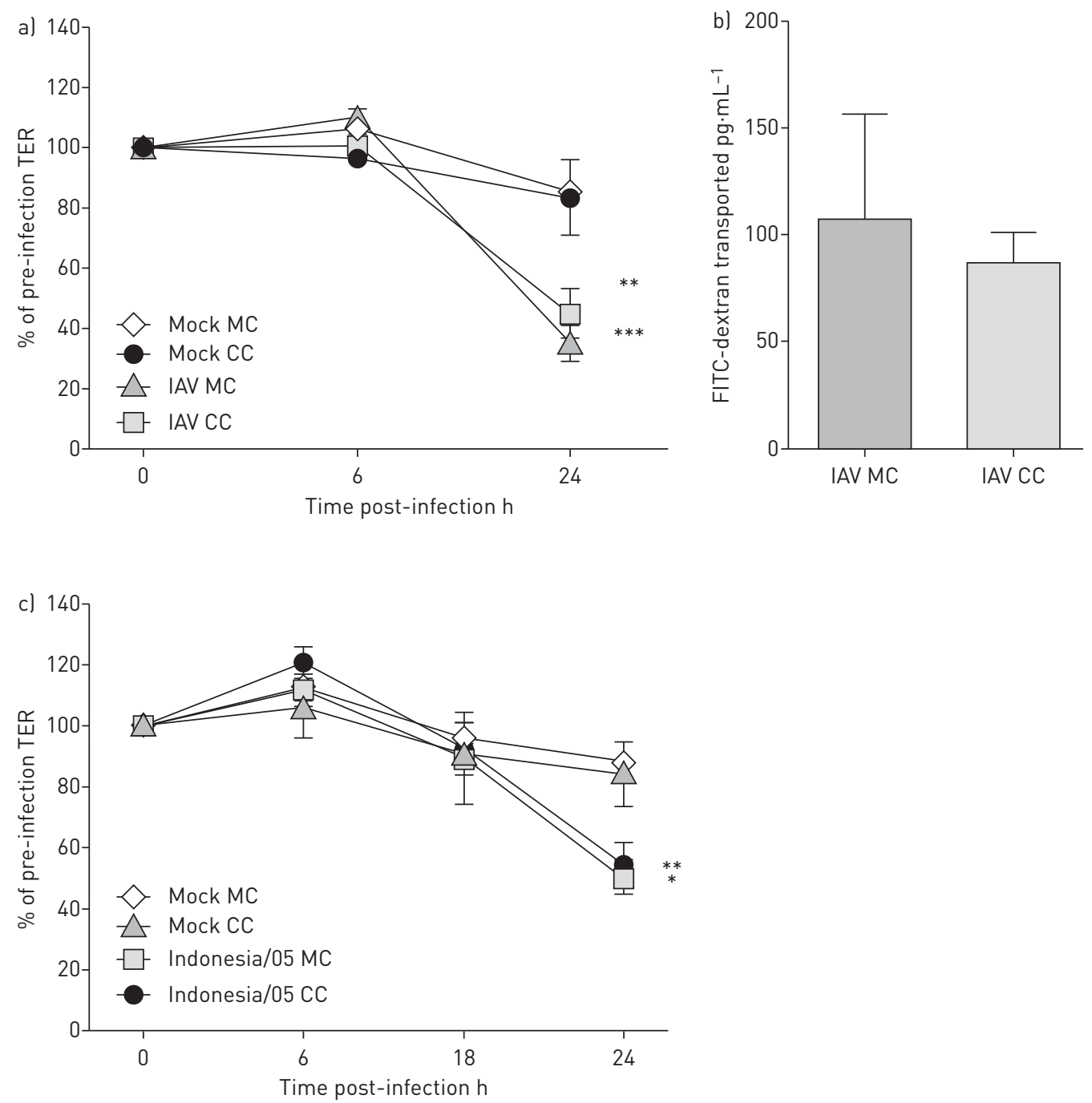

FIGURE 5 Influenza A virus (IAV)-induced damage is independent of endothelial cells. a) Measurement of the trans-barrier electrical resistance (TER) of epithelial-endothelial co-cultures (CC) and epithelial monocultures (MC) following infection with medium ("mock") or IAV (strain PR8/34 (H1N1), at a multiplicity of infection (MOI) of 0.2). b) Permeability of $\mathrm{CC}$ and $\mathrm{MC}$ to fluorescein isothiocyanate (FITC)-dextran following infection with IAV (strain PR8/34, MOI 0.2). Data show the concentrations of FITC detected in the lower compartment following the addition of $2 \mu \mathrm{g}$ of FITC-dextran to the upper compartment. FITC-dextran was added to the upper compartment at $24 \mathrm{~h}$ post-infection and cells were incubated for $3 \mathrm{~h}$ prior to the measurement of FITC in the lower compartment. The data have been mock-subtracted. c) Measurement of the TER of CC and MC following infection with medium ("mock") or IAV strain Indonesia/05 (H5N1). All data are pooled from at least three independent experiments and shown as mean \pm SEM. a and c) Data are expressed relative to the TER recorded just prior to infection (which was defined as $100 \%$ ), and statistical significance relative to mock-infected cells was calculated by two-way ANOVA. ${ }^{*}: p<0.05 ;{ }^{* *}: p<0.01 ;{ }^{* * *}: p<0.001$.

(figure 5c). Together, these data suggest that any soluble factors produced by endothelial cells during IAV infection do not play a significant role in in vitro barrier damage.

\section{Damage to the in vitro alveolar barrier is associated with damage to the epithelial cell tight junction}

We then sought to determine which features of the epithelial cell layer were being damaged by IAV infection. IAV infection of epithelial cells can induce cell death, either by necrosis or apoptosis [3, 4, 30-32]. Thus, we reasoned that the observed barrier damage could simply reflect IAV-induced epithelial cell death. To test this hypothesis, IAV-infected and mock-infected epithelial cells were processed for electron microscopy and examined for morphological changes (figure 6a). Both mock- and IAV-infected cells displayed similar ultrastructural features. Indeed, the only obvious difference between the two treatment groups was that the IAV-infected cells contained particles that were morphologically consistent with influenza virions. To confirm 
a)

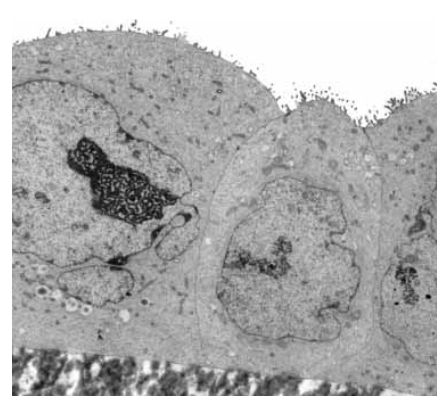

IAV

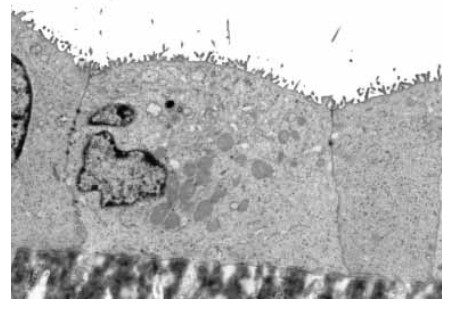

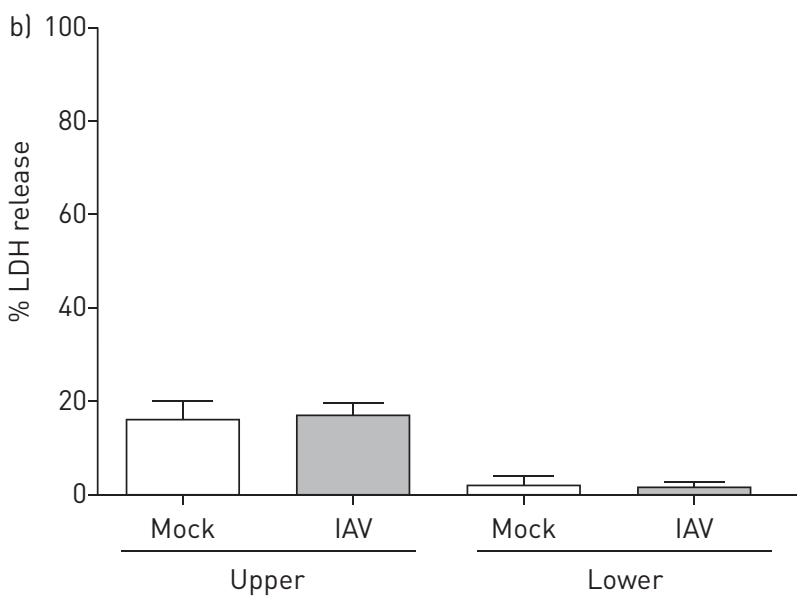

FIGURE 6 Influenza A virus (IAV) does not induce extensive epithelial cell death. Epithelial cells were grown on a transwell membrane and infected with either medium ("mock") or IAV (strain PR8/34 (H1N1), at a multiplicity of infection of 0.2). a) Representative electron microscopy images of epithelial cell monolayers $24 \mathrm{~h}$ after IAV or mock infection. b) Percentage lactate dehydrogenase (LDH) release of epithelial cell monolayers $24 \mathrm{~h}$ after IAV or mock infection. LDH levels were measured in the supernatants from the upper and lower compartments of the transwell, and percentage LDH release was calculated according to the manufacturer's instructions. Data are pooled from three independent experiments and Shown as mean \pm SEM.

the absence of widespread cell death, the release of cytoplasmic LDH into the cell culture medium was also measured $24 \mathrm{~h}$ after IAV infection (figure 6b). No significant difference was observed in LDH release by epithelial cells grown on a transwell membrane, following mock or IAV infection. Taken together, these data suggest that epithelial cell death is unlikely to account for the in vitro barrier damage following IAV infection.

We then reasoned that, like some other respiratory viruses [5], IAV may damage the AJC of pulmonary epithelial cells. We therefore used electron microscopy and the electron-dense tracer lanthanum to measure AJC integrity after IAV infection. Specifically, the number of lanthanum-positive (i.e. damaged) intercellular junctions following IAV or mock infection was determined (figure 7a). IAV infection resulted in significantly more lanthanum-positive epithelial intercellular junctions compared with mock infection, suggesting that IAV infection damages the AJC. Immunofluorescence staining for six different AJC proteins was then performed (figure 7b). No obvious differences in zona occludin-1, $\beta$-catenin and E-cadherin staining were observed between IAV- and mock-infected cells. In contrast, there was a trend towards reduced expression of occludin and junctional adhesion molecule following IAV infection. However, what was most striking was the clear loss of staining of the tight junction claudin-4 following IAV infection. To confirm that the same phenotype could be observed following infection with a highly pathogenic avian influenza virus, epithelial cells infected with Indonesia/05 were also stained for claudin- 4 . Similar to PR8/34 infection, infection with Indonesia/05 resulted in a dramatic loss of claudin-4 staining (figure 7c). Taken together, these data suggest that IAV damages the pulmonary epithelial-endothelial barrier in vitro by damaging the epithelial tight junction, in particular claudin- 4 .

\section{IAV-induced barrier damage occurs independently of cytokines present in the supernatant}

Numerous studies have shown that pro-inflammatory cytokines have a detrimental effect on the integrity of the AJC [33]. We therefore sought to determine whether the observed damage to the AJC was a result of the cytokines produced by epithelial cells upon infection with IAV. Accordingly, the supernatant of IAV-infected and mock-infected co-cultures was harvested at $24 \mathrm{~h}$ post-infection. The supernatant of IAV-infected cells was then treated with an anti-IAV antibody (to prevent re-infection with IAV) and transferred to uninfected epithelial cells. Treatment with IAV supernatant did not result in significant decline in the TER over time compared with treatment with mock supernatant (figure 8a). Similarly, treatment with IAV supernatant did not increase permeability to FITC-labelled dextran (figure 8b). These data therefore suggest that neither cytokines nor any other soluble factors present in the cell supernatant after IAV infection contribute to the observed barrier damage.

\section{Discussion}

Severe IAV infection can damage the alveolar epithelial-endothelial barrier, resulting in pulmonary oedema and respiratory dysfunction. Both epithelial and endothelial cells have been ascribed roles in this damage, although the exact mechanisms remain unclear. 
a)
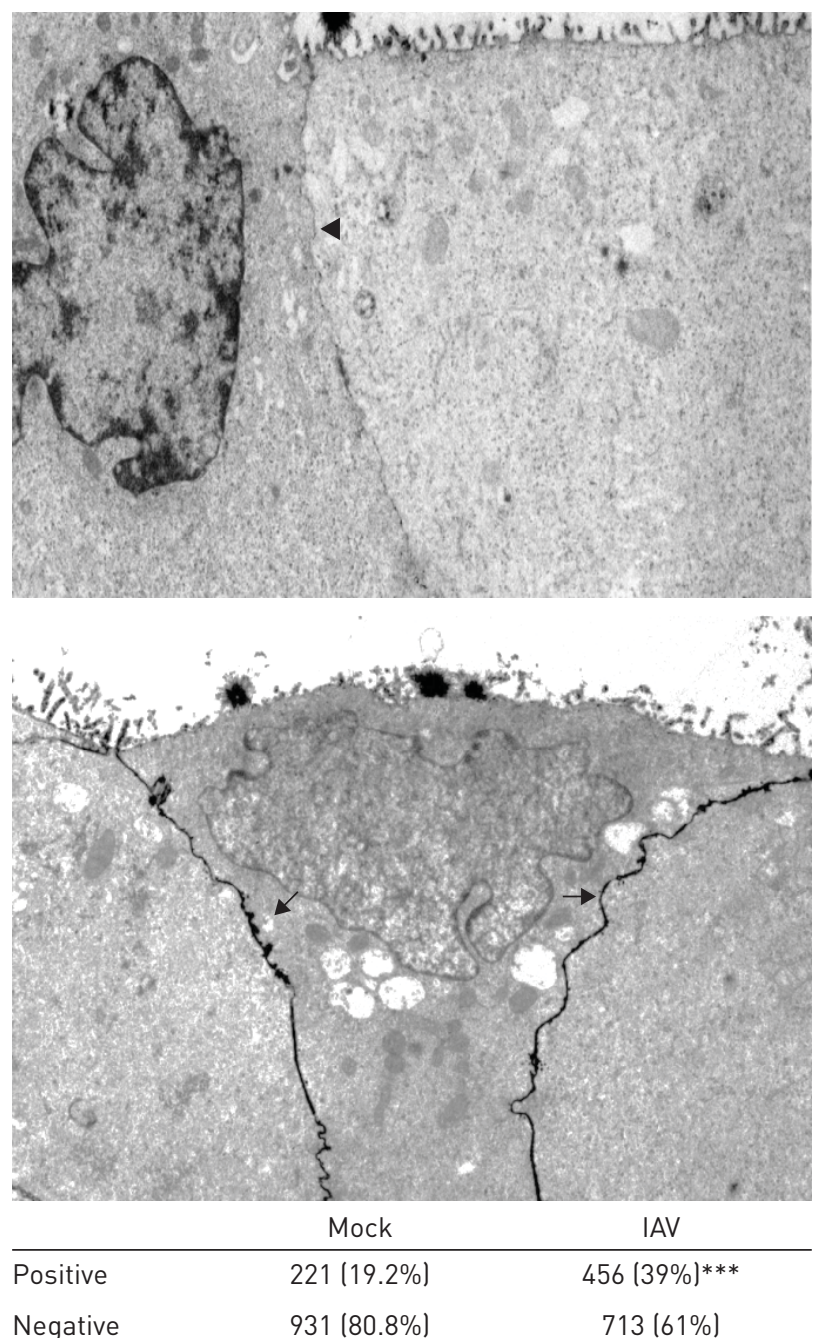

Negative

$931(80.8 \%)$

$713(61 \%)$ b)
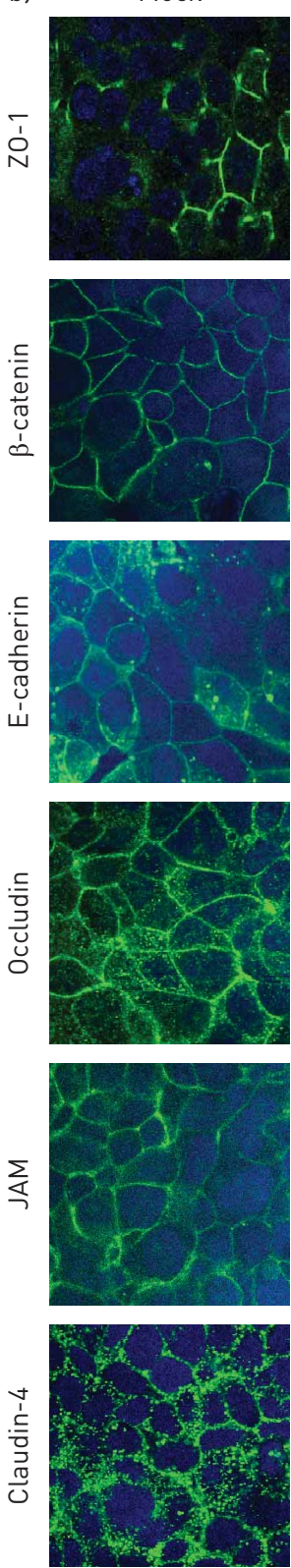

IAV
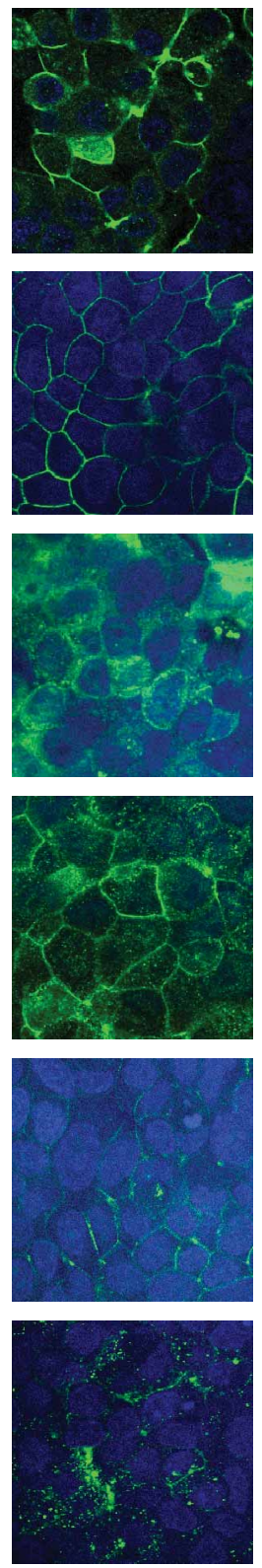
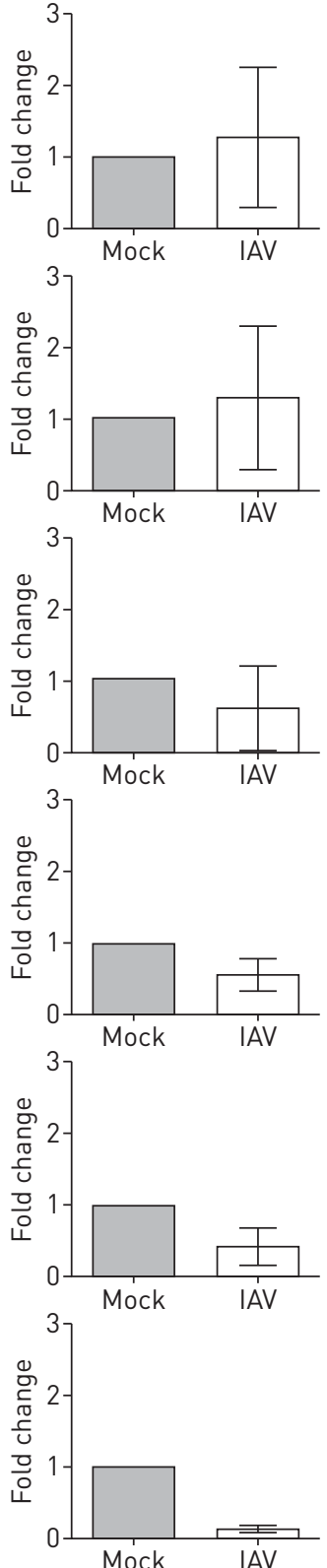
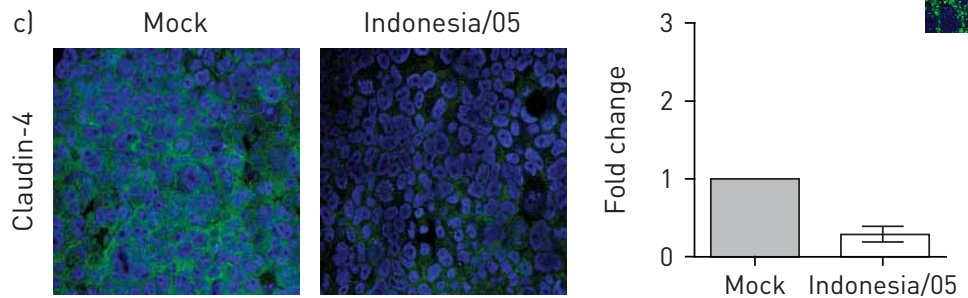

FIGURE 7 Influenza A virus (IAV) damages epithelial cell tight junctions. a) Representative images of epithelial cells treated with lanthanum. Epithelial cells were grown on a transwell membrane and infected with either medium ("mock") or IAV (strain PR8/34 (H1N1), at a multiplicity of infection (MOI) of 0.2). At $24 \mathrm{~h}$ post-infection, cells were fixed (with lanthanum added to the upper compartment), processed for electron microscopy and the number of lanthanum-positive intercellular junctions were counted. An arrowhead indicates an intercellular junction that was considered lanthanum-negative whereas arrows indicate two intercellular junctions that were considered lanthanum-positive. Data are pooled from two independent experiments and the number of cell junctions and relative percentages are shown. Statistical significance was calculated by Fischer's exact test. ${ }^{* * *}: p<0.001 . b$ and $\left.c\right)$ Representative immunofluorescence images of apical junction complex proteins of epithelial cells. Epithelial cells were grown on a transwell membrane and infected with either medium ("mock") or IAV, strains b) PR8/34 (MOI 0.2) or c) Indonesia/05 (H5N1). At $24 \mathrm{~h}$ post-infection, cells were fixed and the nucleus and the relevant apical junction complex protein were stained (blue and green, respectively). The fold changes in fluorescence intensity in IAV-infected cells relative to mock-infected cells are also shown. Data are pooled from b) at least two or c) three independent experiments and shown as mean士SEM. Z0-1: zona occludin-1; JAM: junctional adhesion molecule. 

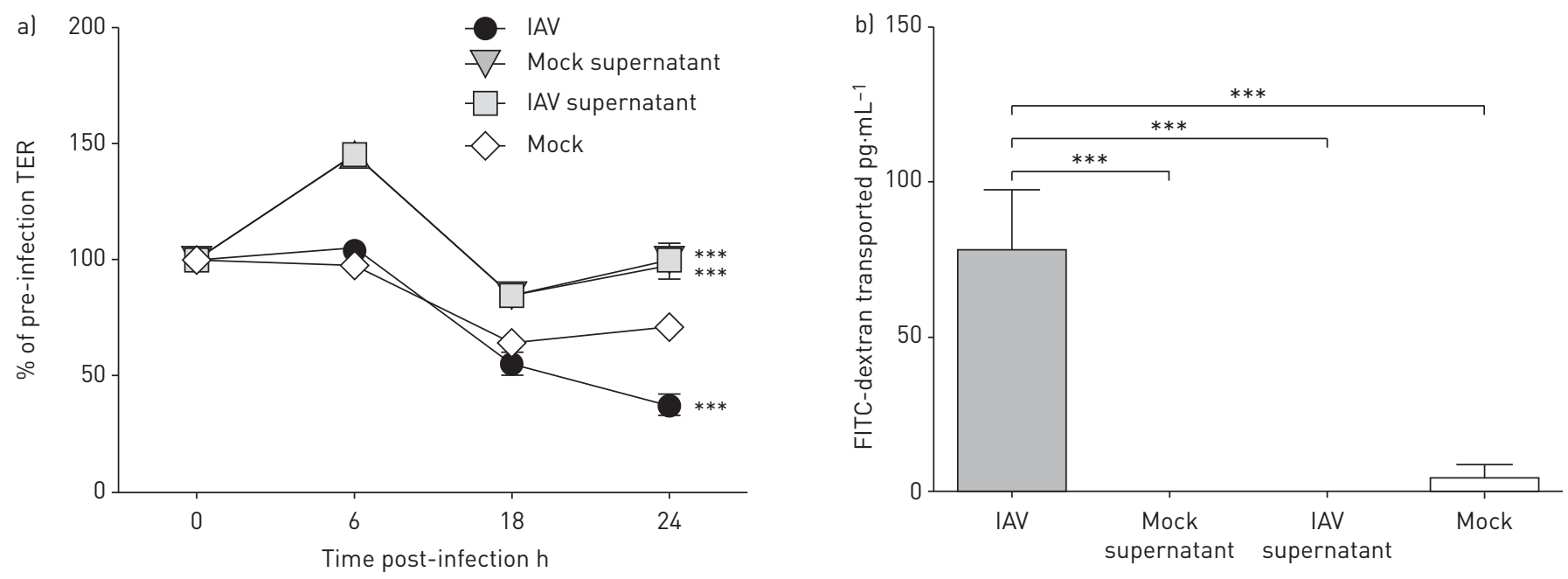

FIGURE 8 Cytokines present in the supernatant do not contribute to the observed barrier damage. a) Measurement of the trans-barrier electrical resistance (TER) of epithelial-endothelial co-cultures following treatment with medium ("mock"), supernatant derived from influenza A virus (IAV)-infected co-cultures (strain PR8/34 (H1N1), at a multiplicity of infection (MOI) of 0.2) at 24 h post-infection ("IAV supernatant"), supernatant derived from mock-infected co-cultures at $24 \mathrm{~h}$ post-infection ("mock supernatant") or IAV infection (PR8/34, MOI 0.2). IAV supernatant was treated with an antibody against IAV (see Materials and methods section) to prevent re-infection with IAV. Data are expressed relative to the TER recorded just prior to infection (which was defined as 100\%). b) Permeability of epithelial-endothelial co-cultures to fluorescein isothiocyanate (FITC)-dextran. Data show the concentrations of FITC detected in the lower compartment following the addition of $2 \mu \mathrm{g}$ of FITC-dextran to the upper compartment. FITC-dextran was added to the upper compartment $24 \mathrm{~h}$ after treatment with medium ("mock"), IAV supernatant, mock supernatant or IAV infection. Cells were incubated for $3 \mathrm{~h}$ prior to the measurement of FITC in the lower compartment. All data are pooled from two independent experiments using the supernatant derived from four independent experiments, and shown as mean \pm SEM. Statistical significance relative to mock-infected cells was calculated by al two-way ANOVA or b) one-way ANOVA with Bonferroni's multiple comparison test. ${ }^{* * *}$ : $p<0.001$.

Here, we used an in vitro model of the epithelial-endothelial barrier to explore the relative roles of each of these two cell types during IAV-induced barrier damage [25]. This reductionist approach is somewhat limited by the fact that it is impossible for an in vitro model to ever completely reflect the complex interactions that happen in the lung during IAV infection. Indeed, this model most likely reflects the situation very early on during infection, when there is not an abundance of leukocytes present in the lung (the presence of which may also have an important impact on barrier integrity). Nevertheless, this model does afford the researcher the unique opportunity to examine both epithelial and endothelial cells in a system more akin to the in vivo situation, i.e. whereby endothelial cells and alveolar epithelial cells are closely apposed and only separated by a basement membrane.

The present study demonstrated that, while IAV damaged the epithelial-endothelial barrier in vitro, this was not associated with endothelial cell infection. This was somewhat surprising, because numerous other in vitro studies to date have shown that monocultures of endothelial cells are susceptible to IAV infection $[8,19,21,22]$. Indeed, we also found that endothelial cells were permissive to IAV infection in the absence of epithelial cells. However, when the epithelial-endothelial co-culture was inoculated with IAV from the epithelial side, infection of endothelial cells was not observed. Given the limited number of virus particles present in the lower compartment of the transwell system, the most likely explanation for this observation is that new virus particles were being released apically from epithelial cells and hence rarely came into contact with the adjacent endothelial cells. This is consistent with previous studies showing that IAV is predominantly released from the apical side of primary human type I pneumocytes [19]. Alternatively, the initial IAV infection in the epithelial cells may allow the closely apposed endothelial cells to become refractory to infection (e.g. via the production of type I interferons). Importantly, in vivo infection of endothelial cells by IAV is rare $[23,24,34,35]$. It is therefore tempting to speculate that, in vivo, epithelial cells serve to limit endothelial cell infection in much the same manner as has been described here.

Murine studies have identified endothelial cells as the key producers of pro-inflammatory cytokines in the lung during IAV infection [11]. We thus reasoned that endothelial cells in vitro, despite not being infected by IAV, could produce cytokines that may play an important role in barrier damage. However, although endothelial cells showed a pro-inflammatory and pro-coagulant response, they did not contribute to the observed barrier damage. One possible explanation for this observation is that endothelial cell-derived cytokines may only be able to damage the alveolar barrier in the presence of leukocytes. For example, CCL8 (which we found to be differentially expressed in endothelial but not epithelial cells) can act as a potent chemoattractant for human monocytes [36]. Given that (monocyte-derived) macrophages are known to play an important role in damaging pulmonary epithelial cells during IAV infection [37], this is 
one possible mechanism by which endothelial-derived cytokines could contribute to barrier damage in vivo but not in vitro.

Here we demonstrated, for the first time, that IAV damaged tight junctions, and specifically claudin-4, of respiratory epithelial cells. Within the alveolus, claudin-4 is expressed by both type I and type II pneumocytes [38]. The importance of claudin-4 in lung function is demonstrated by the fact that claudin-4 knockout mice display increased permeability to 5-carboxyfluorescein and decreased alveolar fluid clearance [39]. In vitro, the transduction of cells with claudin-4 increased the TER by $~ 50 \%$ [40]. Moreover, several different viruses are known to disrupt claudin expression and increase permeability across monolayers of several different cell types [6-8]. It is therefore tempting to speculate that IAV-induced loss of claudin-4 played a major role in the observed decline of the TER. Interestingly, claudin-4 expression is thought to be more sensitive than other proteins of the AJC to changes in the cell or microenvironment [38]. Therefore, it may be possible that a reduction in other proteins of the AJC would be observed at a time-point later than the 24 -h time-point used in the present study. Nevertheless, these data clearly demonstrate that IAV can damage the tight junctions of pulmonary epithelial cells.

There are several possible mechanisms by which IAV could damage the AJC of epithelial cells. Despite evidence that pro-inflammatory cytokines can decrease the TER of epithelial cells and damage the AJC [33], extracellular cytokines produced during IAV infection did not induce barrier damage in our in vitro model. These data suggest that damage to the AJC was the direct result of the virus infection itself, as has been previously described in MDCK cells [9]. Specifically, GoLEBIEWsKi et al. [9] showed that an ESEV consensus motif in the carboxyl terminus of the nonstructural protein 1 (NS-1) of some highly pathogenic H5N1 IAV strains bound to and affected the localisation of select host proteins with a PDZ domain. Some of these PDZ proteins (such as Dlg1 (discs large homolog 1)) are essential in tight junction formation and, thus, influenza virus was able to disrupt the formation of the AJC. Interestingly, claudins are also able to bind PDZ proteins such as Dlg1 [41]. It is therefore tempting to speculate that components of IAV are able to indirectly disrupt the expression of claudins by binding to and sequestering their cognate binding partners.

Taken together, this study shows that the alveolar epithelium both forms the main barrier against fluid leakage to the alveolar lumen and is the key target of IAV upon infection via the respiratory route. This implies that improving and restoring the integrity of the alveolar epithelium may play an important role in reducing the severity of IAV-induced oedema and ARDS. This remains an important area for future studies, where it is hoped that increasingly complex in vitro models (e.g. a triple culture of epithelial cells, endothelial cells and macrophages), combined with in vivo data, will help determine the effect of specific intervention strategies on the restoration of alveolar epithelial cells and respiratory function.

\section{References}

1 Gorin AB, Stewart PA. Differential permeability of endothelial and epithelial barriers to albumin flux. J Appl Physiol Respir Environ Exerc Physiol 1979; 47: 1315-1324.

2 Short KR, Kroeze EJ, Fouchier RA, et al. Pathogenesis of influenza-induced acute respiratory distress syndrome. Lancet Infect Dis 2014; 14: 57-69.

3 Mauad T, Hajjar LA, Callegari GD, et al. Lung pathology in fatal novel human influenza A (H1N1) infection. Am J Respir Crit Care Med 2010; 181: 72-79.

4 Uiprasertkul M, Kitphati R, Puthavathana P, et al. Apoptosis and pathogenesis of avian influenza A (H5N1) virus in humans. Emerg Infect Dis 2007; 13: 708-712.

5 Sajjan U, Wang Q, Zhao Y, et al. Rhinovirus disrupts the barrier function of polarized airway epithelial cells. Am J Respir Crit Care Med 2008; 178: 1271-1281.

$6 \mathrm{Xu} \mathrm{Z}$, Waeckerlin $\mathrm{R}$, Urbanowski MD, et al. West Nile virus infection causes endocytosis of a specific subset of tight junction membrane proteins. PLoS One 2012; 7: e37886.

7 Agrawal T, Sharvani V, Nair D, et al. Japanese encephalitis virus disrupts cell-cell junctions and affects the epithelial permeability barrier functions. PLoS One 2013; 8: e69465.

8 Armstrong SM, Wang C, Tigdi J, et al. Influenza infects lung microvascular endothelium leading to microvascular leak: role of apoptosis and claudin-5. PLoS One 2012; 7: e47323.

9 Golebiewski L, Liu H, Javier RT, et al. The avian influenza virus NS1 ESEV PDZ binding motif associates with Dlg1 and Scribble to disrupt cellular tight junctions. J Virol 2011; 85: 10639-10648.

10 Short KR, Veldhuis Kroeze EJ, Reperant LA, et al. Influenza virus and endothelial cells: a species specific relationship. Front Microbiol 2014; 5: 653.

11 Teijaro JR, Walsh KB, Cahalan S, et al. Endothelial cells are central orchestrators of cytokine amplification during influenza virus infection. Cell 2011; 146: 980-991.

12 Armstrong SM, Darwish I, Lee WL. Endothelial activation and dysfunction in the pathogenesis of influenza A virus infection. Virulence 2013; 4: 537-542.

13 Cheung CY, Poon LL, Lau AS, et al. Induction of proinflammatory cytokines in human macrophages by influenza A (H5N1) viruses: a mechanism for the unusual severity of human disease? Lancet 2002; 360: 1831-1837.

14 Coyne CB, Vanhook MK, Gambling TM, et al. Regulation of airway tight junctions by proinflammatory cytokines. Mol Biol Cell 2002; 13: 3218-3234.

15 Zelaya H, Tada A, Vizoso-Pinto MG, et al. Nasal priming with immunobiotic Lactobacillus rhamnosus modulates inflammation-coagulation interactions and reduces influenza virus-associated pulmonary damage. Inflamm Res 2015; 64: 589-602 

experimental influenza in ferrets. BMC Microbiol 2014; 14: 134.

Bevilacqua MP, Pober JS, Majeau GR, et al. Recombinant tumor necrosis factor induces procoagulant activity in cultured human vascular endothelium: characterization and comparison with the actions of interleukin 1. Proc Natl Acad Sci USA 1986; 83: 4533-4537.

18 de Jonge E, Friederich PW, Vlasuk GP, et al. Activation of coagulation by administration of recombinant factor VIIa elicits interleukin 6 (IL-6) and IL-8 release in healthy human subjects. Clin Diagn Lab Immunol 2003; 10: 495-497.

19 Chan MC, Chan RW, Yu WC, et al. Influenza H5N1 virus infection of polarized human alveolar epithelial cells and lung microvascular endothelial cells. Respir Res 2009; 10: 102.

20 Ishiguro $\mathrm{N}$, Takada $\mathrm{A}$, Yoshioka $\mathrm{M}$, et al. Induction of interferon-inducible protein-10 and monokine induced by interferon- $\gamma$ from human endothelial cells infected with influenza A virus. Arch Virol 2004; 149: 17-34.

21 Ocaña-Macchi M, Bel M, Guzylack-Piriou L, et al. Hemagglutinin-dependent tropism of H5N1 avian influenza virus for human endothelial cells. J Virol 2009; 83: 12947-12955.

22 Zeng H, Pappas C, Belser JA, et al. Human pulmonary microvascular endothelial cells support productive replication of highly pathogenic avian influenza viruses: possible involvement in the pathogenesis of human $\mathrm{H} 5 \mathrm{~N} 1$ virus infection. J Virol 2012; 86: 667-678.

23 Shieh WJ, Blau DM, Denison AM, et al. 2009 pandemic influenza A (H1N1): pathology and pathogenesis of 100 fatal cases in the United States. Am J Pathol 2010; 177: 166-175.

24 Kuiken T, Taubenberger JK. Pathology of human influenza revisited. Vaccine 2008; 26: Suppl. 4, D59-D66.

25 Kasper J, Hermanns MI, Bantz C, et al. Inflammatory and cytotoxic responses of an alveolar-capillary coculture model to silica nanoparticles: comparison with conventional monocultures. Part Fibre Toxicol 2011; 8: 6.

26 Salomon JJ, Muchitsch VE, Gausterer JC, et al. The cell line NCl-H441 is a useful in vitro model for transport studies of human distal lung epithelial barrier. Mol Pharm 2014; 11: 995-1006.

27 Unger RE, Krump-Konvalinkova V, Peters K, et al. In vitro expression of the endothelial phenotype: comparative study of primary isolated cells and cell lines, including the novel cell line HPMEC-ST1.6R. Microvasc Res 2002; 64: 384-397.

28 de Wit E, Spronken MI, Bestebroer TM, et al. Efficient generation and growth of influenza virus A/PR/8/34 from eight cDNA fragments. Virus Res 2004; 103: 155-161.

29 Goeijenbier M, Meijers JC, Anfasa F, et al. Effect of Puumala hantavirus infection on human umbilical vein endothelial cell hemostatic function: platelet interactions, increased tissue factor expression and fibrinolysis regulator release. Front Microbiol 2015; 6: 220.

30 Brydon EW, Smith H, Sweet C. Influenza A virus-induced apoptosis in bronchiolar epithelial (NCI-H292) cells limits pro-inflammatory cytokine release. J Gen Virol 2003; 84: 2389-2400.

31 Arndt U, Wennemuth G, Barth P, et al. Release of macrophage migration inhibitory factor and CXCL8/ interleukin-8 from lung epithelial cells rendered necrotic by influenza A virus infection. J Virol 2002; 76: 9298-9306.

32 Lam WY, Tang JW, Yeung AC, et al. Avian influenza virus A/HK/483/97 (H5N1) NS1 protein induces apoptosis in human airway epithelial cells. J Virol 2008; 82: 2741-2751.

33 Capaldo CT, Nusrat A. Cytokine regulation of tight junctions. Biochim Biophys Acta 2009; 1788: 864-871.

$34 \mathrm{Gu}$ J, Xie Z, Gao Z, et al. H5N1 infection of the respiratory tract and beyond: a molecular pathology study. Lancet 2007; 370: 1137-1145.

35 Kuiken T, van den Brand J, van Riel D, et al. Comparative pathology of select agent influenza A virus infections. Vet Pathol 2010; 47: 893-914.

36 Uguccioni M, D’Apuzzo M, Loetscher M, et al. Actions of the chemotactic cytokines MCP-1, MCP-2, MCP-3, RANTES, MIP- $1 \alpha$ and MIP-1 $\beta$ on human monocytes. Eur J Immunol 1995; 25: 64-68.

37 Herold S, Steinmueller M, von Wulffen W, et al. Lung epithelial apoptosis in influenza virus pneumonia: the role of macrophage-expressed TNF-related apoptosis-inducing ligand. J Exp Med 2008; 205: 3065-3077.

38 Overgaard CE, Mitchell LA, Koval M. Roles for claudins in alveolar epithelial barrier function. Ann NY Acad Sci 2012; 1257: 167-174.

39 Kage H, Flodby P, Gao D, et al. Claudin 4 knockout mice: normal physiological phenotype with increased susceptibility to lung injury. Am J Physiol Lung Cell Mol Physiol 2014; 307: L524-L536.

40 Mitchell LA, Overgaard CE, Ward C, et al. Differential effects of claudin-3 and claudin-4 on alveolar epithelial barrier function. Am J Physiol Lung Cell Mol Physiol 2011; 301: L40-L49.

41 Brennan K, Offiah G, McSherry EA, et al. Tight junctions: a barrier to the initiation and progression of breast cancer? J Biomed Biotechnol 2010; 2010: 460607. 